\title{
Brazilian Portuguese version of the Anger Rumination Scale (ARS-Brazil)
}

\author{
Versão em português brasileiro da Escala de Ruminação de Raiva (ARS-Brasil)
}

Daniela Sperotto, ${ }^{1}$ Arthur Gus Manfro, ${ }^{1}$ Luiza Kvitko Axelrud, ${ }^{1}$ Pedro Henrique Manfro, ${ }^{2}$ Giovanni Abrahão Salum, ${ }^{1}$ Diogo Araújo DeSousa ${ }^{1}$

\begin{abstract}
Objective: To describe the cross-cultural adaptation of the Anger Rumination Scale (ARS) for use in Brazil.

Methods: The cross-cultural adaptation followed a four-step process, based on specialized literature: 1) investigation of conceptual and item equivalence; 2) translation and backtranslation; 3) pretest; and 4) investigation of operational equivalence.

Results: A final Brazilian version of the instrument (ARS-Brazil) was defined and is presented. Pretest results revealed that the instrument was generally well understood by adults as well as indicated a few modifications that were included in the final version presented here.

Conclusion: The Brazilian Portuguese version of the ARS seems to be very similar to the original ARS in terms of conceptual and item equivalence, semantics, and operational equivalence, suggesting that future cross-cultural studies may benefit from this early version. As a result, a new instrument is now available for the assessment of rumination symptoms of anger and irritability for adults in community, clinical, and research settings.

Keywords: Rumination, anger, irritability, anger rumination scale.
\end{abstract}

\section{Resumo}

Objetivo: Descrever a adaptação transcultural da Escala de Ruminação de Raiva (Anger Rumination Scale, ARS) para uso no Brasil.

Método: A adaptação transcultural seguiu um processo de quatro etapas baseado em literatura especializada: 1 ) investigação da equivalência conceitual e dos itens; 2) tradução e retrotradução; 3) pré-teste; e 4) investigação da equivalência operacional.

Resultados: Uma versão final brasileira do instrumento, denominada ARS-Brasil, foi obtida e é apresentada. Os resultados do pré-teste demonstraram que a escala foi predominantemente bem entendida entre adultos, e indicaram algumas modificações que foram incluídas na versão final.

Conclusão: A versão da ARS adaptada para o português brasileiro mostra-se muito similar à versão original da ARS no que diz respeito à equivalência conceitual e dos itens, semântica e equivalência operacional, sugerindo que futuros estudos transculturais poderiam se beneficiar desta primeira versão. Como resultado, um novo instrumento está agora disponível para a avaliação de sintomas de ruminação da raiva e da irritabilidade para adultos, em contextos comunitário, clínico e de pesquisa. Descritores: Ruminação, raiva, irritabilidade, escala de ruminação de raiva.

\footnotetext{
${ }^{1}$ Seção de Afeto Negativo e Processos Sociais, Departamento de Psiquiatria, Hospital de Clínicas de Porto Alegre (HCPA), Universidade Federal do Rio Grande do Sul (UFRGS), Porto Alegre, RS, Brazil. 2 Pontifícia Universidade Católica do Rio Grande do Sul (PUCRS), Porto Alegre, RS, Brazil. Submitted Mar 06 2017, accepted for publication Jul 172017.

Suggested citation: Sperotto D, Manfro AG, Axelrud LK, Manfro PH, Salum GA, DeSousa DA. Brazilian Portuguese version of the Anger Rumination Scale (ARS-Brazil). Trends Psychiatry Psychother. 2018;40(1):8-15. http://dx.doi.org/10.1590/2237-6089-2017-0026
} 


\section{Introduction}

Anger is a basic emotion characterized by a state of negative unpleasant feelings, specific cognitive assessments and biased action and behavior., ${ }^{1,2}$ Anger rumination is regarded as a relatively independent component within the larger sequence of anger phenomenology, and can be defined as conscious thoughts about the experience of anger occurring without the demands of the immediate situation. ${ }^{2-4}$ The Anger Rumination Scale (ARS) was developed to assess this construct. ${ }^{2}$ This is important because the tendency to ruminate about anger may be partly responsible for maintaining anger as a trait, and therefore it may be related to several negative health outcomes associated with trait anger. ${ }^{5}$

The ARS was built to measure the tendency to focus on the angry mood, to remember past episodes of anger, and to think about the causes and consequences of episodes of anger. Authors suggest that memories of past episodes of anger can trigger new episodes of state-angry; attention to anger experiences can lead to an amplification of its intensity and duration; and counterfactual thoughts can be related to a tendency to retaliation. The term "counterfactual thinking" refers to cognitions about antecedents and consequences of episodes of anger. ${ }^{2}$ Previous psychometric studies using the ARS have shown that the instrument has adequate internal consistency (Cronbach's alpha of 0.93). Factor analysis revealed four subscales: angry afterthoughts, thoughts of revenge, angry memories and understanding the causes, all with Cronbach's alpha values ranging from 0.77 to 0.86 , with item total correlations ranging from 0.39 to 0.75 . Further analyses also revealed a good test-retest reliability of 0.77 over a 1-month period and good concurrent validity with other measures of anger, negative affectivity and the individual's ability to reflect on emotions. ${ }^{2}$ The psychometric properties of the ARS were evaluated in validations in other countries, and the factorial structure (four factors) of the original scale was also observed in most of the studies. For example, in the validation study of Mexico, the four-factor model had better goodness of fit indices than rival models with three and two factors. Alpha reliabilities were acceptable (0.72-0.89). ${ }^{3}$ In the Australian sample, the four-factor model provided a good fit: SatorraBentler chi-square (S-B $X^{2}$ ) (degrees of freedom [df] $=146)=489.48, p<0.001$; root mean square error of approximation (RMSEA) $=0.06$ ( $90 \%$ confidence interval $[90 \% \mathrm{CI}]=0.06-0.07)$; comparative fit index $(C F I)=0.91$; standardized root-mean-square residual $(\mathrm{SRMR})=0.05 .^{6}$
Despite its importance, anger rumination has received little attention in the Brazilian literature. One potential reason is that scales such as the ARS are not available in a validated form to the Brazilian population. Although studies in this matter have mostly concentrated in the United States, other countries and populations have successfully adapted the ARS to their languages and cultural contexts, such as the Spanish, ${ }^{7}$ the Mexicans ${ }^{3}$ and the Iranians, ${ }^{8}$ among others. $6,9,10$ The overall good factor structure properties of the original scale and its cross-cultural adaptations demonstrate that Brazilian researchers would benefit from a cross-cultural adaptation that would make this instrument available as a possibility to investigate anger rumination in our populational context. Therefore, the objective of the present study was to conduct and describe the process of crosscultural adaptation of the ARS for use in Brazil.

\section{Method}

The study protocol was approved by the ethics committee of Hospital de Clínicas de Porto Alegre (HCPA), Universidade Federal do Rio Grande do Sul (UFRGS; project 15-0032). Translators and collaborators who rated the clarity of the items verbally assented to participate in the study.

\section{Instrument: Anger Rumination Scale (ARS)}

The ARS was developed to measure the "tendency to think about current anger-provoking situations and to recall anger episodes from the past."2 In each item, respondents are instructed to check the word that best describes how often they experience the behaviors, feelings, and reactions described in the scale (reflecting angry symptoms and/or anger rumination symptoms). In the original study, factor analysis results revealed the presence of four factors or subscales: 1) angry afterthoughts (six items); 2) thoughts of revenge (four items); 3) angry memories (five items); and 4) understanding of causes (four items). A four-point word scale is used: almost never, sometimes, often, almost always. For the purposes of this study, participants were asked to answer based on how they felt over the last six months. All items are phrased so that higher scores represent higher levels of anger rumination. Participants are instructed that there are no right or wrong answers when answering to the scale items.

\section{Steps of the cross-cultural adaptation process}

First, the authors of the present study contacted the author of the original ARS so that she could authorize 
the cross-cultural adaptation process. Once permission was granted, a four-step process was followed, based on specialized literature ${ }^{11,12}$ and on the International Test Commission Guidelines for Translating and Adapting Tests. ${ }^{13}$ The four steps were: 1 ) investigation of conceptual and item equivalence; 2) translation and back-translation; 3) pretest; and 4) investigation of operational equivalence.

\section{Investigation of conceptual and item equivalence}

In the first step, the scale was analyzed in terms of conceptual and item equivalence between the original and target contexts. ${ }^{11,12}$ Equivalence was assessed through a literature review about anger rumination and the instruments available for the assessment of anger in Brazil, and based on the analysis and opinion of a committee organized to evaluate the Brazilian version of the ARS (two experts in the field of psychometrics, cited below). The objectives were: 1 ) to investigate if the relationship between the scale and its underlying concept (i.e., anger rumination) in the original setting would be the same in Brazil; and 2) to investigate if the items comprising the original scale would remain relevant and acceptable in the Brazilian context. Instrument analysis was performed by two experts in the field: a researcher with experience in psychometric research (GAS) and a psychologist specialized in crosscultural adaptation of instruments (DADS).

\section{Translation and back-translation}

In the second step, the scale was translated from English into Brazilian Portuguese and then backtranslated into English. Two independent translators produced forward-translations of the ARS, and a third one synthesized both translations into a single version in Brazilian Portuguese. This synthesized version was then back-translated independently by two other translators, and again a third one synthesized both back-translations into a single version in English. ${ }^{11,14,15}$ All translators involved in this step were fluent in both languages: English and Brazilian Portuguese.

The original version of the ARS, the synthesized forward-translation, and thesynthesized back-translation were all evaluated by the same expert committee that assessed conceptual and item equivalence. ${ }^{11,14,15}$ The committee assessed whether the items included in the three versions reflected the same ideas regarding the target construct (i.e., anger rumination). The objective was to make sure that the translation process was adequately conducted and that the translated items were relevant to the Brazilian context. Adjustment of instrument items was performed after consensus was reached among all members of the committee.

\section{Pretest}

The third step of the cross-cultural adaptation process consisted of a pilot study. ${ }^{11,14}$ The aim of this step was to evaluate the understanding of the scale by the target population - Brazilian adults aged 18 years or older. Participants were recruited via e-mail with a link to access the initial version of the scale. The authors contacted graduate students of UFRGS, asking them to share the study link with acquaintances or family members, giving priority to family members/ friends/acquaintances with low socioeconomic status. Forty-nine adults answered the ARS in the pretest. In addition to responding the scale, participants were asked to assess the degree of understanding of each sentence responding to the question "How clear is this sentence?," with the following response options: "I understood it completely," "I understood it more or less" and "I did not understand it." We also asked participants to give suggestions for each of the scale items. Answers were analyzed in an attempt to identify any problems in the wording of the items, as well as any confusing or misleading items.

\section{Investigation of operational equivalence}

In the fourth step, the scale was analyzed in terms of the operational equivalence between the original and target contexts. ${ }^{11,12}$ The following aspects were evaluated considering the use of the instrument in Brazil: instructions, method of administration, questionnaire format, and measurement methods used in the original ARS. Operational equivalence was analyzed through a literature review focusing on operational models of other anger evaluation instruments available in Brazil.

\section{Results}

Results obtained in each step of the adaptation process of the ARS-Brazil are described below (Table 1).

\section{Investigation of conceptual and item equivalence}

Both experts agreed that the domains and theoretical foundation that formed the basis for the original ARS, as well as the items representing them, were equally relevant and important to the target context, and that the actual construction was likely to be equally valid in Brazil. However, both experts identified problems in a specific item of the ARS: "I ruminate about my past anger experiences." In the Brazilian context, the term "ruminate" is not clear to many people as a cognitive process, since it is predominantly used to describe the act of swallowing/regurgitating/chewing/swallowing of a suborder of herbivorous mammals (e.g., cows). 
Table 1 - Translation and back-translation of the ARS to Brazilian Portuguese

\begin{tabular}{|c|c|c|c|c|c|c|}
\hline $\begin{array}{l}\text { Original } \\
\text { ARS }\end{array}$ & $\begin{array}{l}\text { Translation } 1 \\
\text { (BP) }\end{array}$ & $\begin{array}{l}\text { Translation } 2 \\
\text { (BP) }\end{array}$ & $\begin{array}{l}\text { Final } \\
\text { translation (BP) }\end{array}$ & $\begin{array}{l}\text { Back- } \\
\text { translation } 1 \text { (E) }\end{array}$ & $\begin{array}{l}\text { Back- } \\
\text { translation } 2 \text { (E) }\end{array}$ & $\begin{array}{l}\text { Final Back- } \\
\text { translation (E) }\end{array}$ \\
\hline $\begin{array}{l}\text { Anger Rumination } \\
\text { Scale }\end{array}$ & $\begin{array}{l}\text { Escala de } \\
\text { Ruminação Irritada }\end{array}$ & $\begin{array}{l}\text { Escala de } \\
\text { Ruminação Irritada }\end{array}$ & $\begin{array}{l}\text { Escala de } \\
\text { Ruminação Irritada }\end{array}$ & $\begin{array}{l}\text { Anger Rumination } \\
\text { Scale }\end{array}$ & $\begin{array}{l}\text { Anger Rumination } \\
\text { Scale }\end{array}$ & $\begin{array}{l}\text { Anger Rumination } \\
\text { Scale }\end{array}$ \\
\hline $\begin{array}{l}\text { Everyone gets angry } \\
\text { and frustrated } \\
\text { occasionally but } \\
\text { people differ in the } \\
\text { ways that they think } \\
\text { about their episodes } \\
\text { of anger. Statements } \\
\text { below describe } \\
\text { different ways that } \\
\text { people may be } \\
\text { recalling or thinking } \\
\text { about their anger } \\
\text { experiences. Please, } \\
\text { read each statement } \\
\text { and then respond } \\
\text { by circling the } \\
\text { appropriate number } \\
\text { for each statement. } \\
\text { There are no right } \\
\text { or wrong answers in } \\
\text { this questionnaire, } \\
\text { and your honest } \\
\text { responses that best } \\
\text { describe yourself } \\
\text { are very important. } \\
\text { Please, respond to } \\
\text { all items. }\end{array}$ & $\begin{array}{l}\text { Todo mundo se } \\
\text { sente com raiva ou } \\
\text { frustrado às vezes. } \\
\text { Porém, as pessoas } \\
\text { são diferentes com } \\
\text { relação à maneira } \\
\text { de pensar sobre } \\
\text { seus episódios de } \\
\text { raiva. As frases } \\
\text { abaixo descrevem } \\
\text { diferentes maneiras } \\
\text { que as pessoas } \\
\text { podem se lembrar } \\
\text { ou pensar sobre } \\
\text { situações de raiva. } \\
\text { Por favor, leia cada } \\
\text { uma das frases e } \\
\text { circule o número } \\
\text { apropriado para } \\
\text { cada frase. Não } \\
\text { existem respostas } \\
\text { certas ou erradas } \\
\text { nesse questionário, } \\
\text { e a resposta que } \\
\text { melhor Ihe descreve } \\
\text { é muito importante. } \\
\text { Por favor, responda } \\
\text { a todos os itens. }\end{array}$ & $\begin{array}{l}\text { Todo mundo fica com } \\
\text { raiva e frustrado } \\
\text { de vez em quando, } \\
\text { mas o que diferencia } \\
\text { as pessoas são as } \\
\text { formas como elas } \\
\text { pensam sobre os } \\
\text { seus episódios de } \\
\text { raiva. As frases } \\
\text { abaixo descrevem } \\
\text { diferentes formas } \\
\text { as quais as pessoas } \\
\text { relembram ou } \\
\text { pensam sobre } \\
\text { seus episódios de } \\
\text { raiva. Por favor, } \\
\text { leia cada frase e } \\
\text { responda circulando } \\
\text { o número que } \\
\text { melhor representa } \\
\text { cada frase. Não } \\
\text { existem respostas } \\
\text { certas ou erradas } \\
\text { neste questionário, e } \\
\text { respostas honestas } \\
\text { que melhor Ihe } \\
\text { descreva são muito } \\
\text { importantes. Por } \\
\text { favor, responda a } \\
\text { todos os itens. }\end{array}$ & $\begin{array}{l}\text { Todo mundo fica com } \\
\text { raiva e frustrado } \\
\text { de vez em quando, } \\
\text { mas as pessoas } \\
\text { são diferentes em } \\
\text { relação à maneira } \\
\text { de pensar sobre } \\
\text { seus momentos } \\
\text { de raiva. As frases } \\
\text { abaixo descrevem } \\
\text { diferentes maneiras } \\
\text { pelas quais as } \\
\text { pessoas lembram } \\
\text { ou pensam sobre } \\
\text { seus momentos } \\
\text { de raiva. Por favor, } \\
\text { leia cada uma das } \\
\text { frases e circule o } \\
\text { número apropriado } \\
\text { para cada frase. Não } \\
\text { existem respostas } \\
\text { certas ou erradas } \\
\text { neste questionário, } \\
\text { seja sincero quanto } \\
\text { às respostas } \\
\text { que melhor Ihe } \\
\text { descrevem nos } \\
\text { últimos } 6 \text { meses. Por } \\
\text { favor, responda a } \\
\text { todas as frases. }\end{array}$ & $\begin{array}{l}\text { Everybody gets } \\
\text { angry and/ } \\
\text { or frustrated } \\
\text { sometimes, but } \\
\text { people are different } \\
\text { in the way they } \\
\text { think about these } \\
\text { moments of anger } \\
\text { and frustration. } \\
\text { The next sentences } \\
\text { describe the different } \\
\text { ways in which people } \\
\text { remember or think } \\
\text { about their moments } \\
\text { of anger. Please, } \\
\text { read each one of } \\
\text { the sentences and } \\
\text { circle the appropriate } \\
\text { number for each } \\
\text { sentence. There are } \\
\text { no right or wrong } \\
\text { answers in this } \\
\text { questionnaire, so } \\
\text { please be honest in } \\
\text { giving the answers } \\
\text { that best describe } \\
\text { you in the last } 6 \\
\text { months. Please, } \\
\text { respond to all } \\
\text { sentences. }\end{array}$ & $\begin{array}{l}\text { Everybody gets } \\
\text { angry and frustrated } \\
\text { now and then, but } \\
\text { people are different } \\
\text { as to how they } \\
\text { think about their } \\
\text { moments of anger. } \\
\text { The sentences } \\
\text { below describe } \\
\text { different ways which } \\
\text { people remember } \\
\text { or think about their } \\
\text { moments of anger. } \\
\text { Please, read each } \\
\text { of the statements } \\
\text { and circle the } \\
\text { appropriate number } \\
\text { of each one of } \\
\text { them. In this } \\
\text { questionnaire, there } \\
\text { is not an answer } \\
\text { that is right or } \\
\text { wrong, so please } \\
\text { be sincere about } \\
\text { which response best } \\
\text { describes you in } \\
\text { the last } 6 \text { months. } \\
\text { Please, answer all } \\
\text { the questions. }\end{array}$ & $\begin{array}{l}\text { Everybody gets } \\
\text { angry and frustrated } \\
\text { now and then, but } \\
\text { people are different } \\
\text { as to how they } \\
\text { think about their } \\
\text { moments of anger. } \\
\text { The sentences below } \\
\text { describe different } \\
\text { ways which people } \\
\text { remember or think } \\
\text { about their moments } \\
\text { of anger. Please, } \\
\text { read each one of the } \\
\text { sentences and circle } \\
\text { the appropriate } \\
\text { number for each one } \\
\text { of them. There are } \\
\text { no right or wrong } \\
\text { answers in this } \\
\text { questionnaire, so } \\
\text { please be honest in } \\
\text { giving the answers } \\
\text { that best describe } \\
\text { you in the last } 6 \\
\text { months. Please, } \\
\text { answer all the } \\
\text { questions. }\end{array}$ \\
\hline $\begin{array}{l}\text { I. I ruminate } \\
\text { about my } \\
\text { past anger } \\
\text { experiences. }\end{array}$ & $\begin{array}{l}\text { Eu penso muito } \\
\text { sobre minhas } \\
\text { experiências de } \\
\text { raiva que já tive. }\end{array}$ & $\begin{array}{l}\text { Eu rumino sobre as } \\
\text { minhas experiências } \\
\text { de raiva passadas. }\end{array}$ & $\begin{array}{l}\text { Eu penso muito } \\
\text { sobre momentos } \\
\text { passados em que } \\
\text { tive raiva. }\end{array}$ & $\begin{array}{l}\text { I think a lot about } \\
\text { moments in the pass } \\
\text { when I felt anger. }\end{array}$ & $\begin{array}{l}\text { I think a lot about } \\
\text { past moments when } \\
\text { I was angry. }\end{array}$ & $\begin{array}{l}\text { I think a lot about } \\
\text { past moments when } \\
\text { I felt anger. }\end{array}$ \\
\hline $\begin{array}{l}\text { 2. I ponder about } \\
\text { the injustices } \\
\text { that have been } \\
\text { done to me. }\end{array}$ & $\begin{array}{l}\text { Eu fico analisando } \\
\text { situações de } \\
\text { injustiça que foram } \\
\text { cometidas contra } \\
\text { mim. }\end{array}$ & $\begin{array}{l}\text { Eu fico pensando } \\
\text { sobre as injustiças } \\
\text { que já foram feitas } \\
\text { à mim. }\end{array}$ & $\begin{array}{l}\text { Eu fico analisando } \\
\text { as situações de } \\
\text { injustiça que } \\
\text { aconteceram } \\
\text { comigo. }\end{array}$ & $\begin{array}{l}\text { I keep analyzing } \\
\text { the situations of } \\
\text { injustice that have } \\
\text { happened to me. }\end{array}$ & $\begin{array}{l}\text { I analyze unfair } \\
\text { situations that } \\
\text { happened to me. }\end{array}$ & $\begin{array}{l}\text { I keep analyzing } \\
\text { the situations } \\
\text { of injustice that } \\
\text { happened to me. }\end{array}$ \\
\hline $\begin{array}{l}\text { 3. } \text { I keep thinking } \\
\text { about events } \\
\text { that angered } \\
\text { me for a long } \\
\text { time. }\end{array}$ & $\begin{array}{l}\text { Eu penso durante } \\
\text { muito tempo sobre } \\
\text { situações que me } \\
\text { deixaram com a } \\
\text { raiva. }\end{array}$ & $\begin{array}{l}\text { Eu fico pensando } \\
\text { por muito tempo } \\
\text { em eventos que me } \\
\text { chatearam. }\end{array}$ & $\begin{array}{l}\text { Eu fico pensando } \\
\text { por muito tempo } \\
\text { sobre situações que } \\
\text { me deixaram com } \\
\text { raiva. }\end{array}$ & $\begin{array}{l}\text { I keep thinking for a } \\
\text { long time about the } \\
\text { situations that make } \\
\text { me angry. }\end{array}$ & $\begin{array}{l}\text { I think for a } \\
\text { long time about } \\
\text { situations that made } \\
\text { me angry. }\end{array}$ & $\begin{array}{l}\text { I keep thinking for a } \\
\text { long time about the } \\
\text { situations that made } \\
\text { me angry. }\end{array}$ \\
\hline $\begin{array}{l}\text { 4. I have long } \\
\text { living fantasies } \\
\text { of revenge after } \\
\text { the conflict is } \\
\text { over. }\end{array}$ & $\begin{array}{l}\text { Eu crio muitas } \\
\text { fantasias a respeito } \\
\text { das vinganças } \\
\text { que eu poderia } \\
\text { ter depois que a } \\
\text { situação de conflito } \\
\text { já acabou. }\end{array}$ & $\begin{array}{l}\text { Depois que um } \\
\text { conflito termina, } \\
\text { tenho fantasias de } \\
\text { vingança por um } \\
\text { longo tempo. }\end{array}$ & $\begin{array}{l}\text { Depois que um } \\
\text { conflito termina, fico } \\
\text { imaginando formas } \\
\text { de vingança por um } \\
\text { longo tempo. }\end{array}$ & $\begin{array}{l}\text { After a conflict ends, } \\
\text { I keep imagining } \\
\text { different ways to get } \\
\text { revenge for a long } \\
\text { time. }\end{array}$ & $\begin{array}{l}\text { After a conflict } \\
\text { is over, I keep } \\
\text { imagining ways of } \\
\text { revenge for a long } \\
\text { time. }\end{array}$ & $\begin{array}{l}\text { After a conflict } \\
\text { is over, I keep } \\
\text { imagining ways of } \\
\text { revenge for a long } \\
\text { time. }\end{array}$ \\
\hline $\begin{array}{l}\text { 5. I think about } \\
\text { certain events } \\
\text { from a long } \\
\text { time ago and } \\
\text { they still make } \\
\text { me angry. }\end{array}$ & $\begin{array}{l}\text { Eu penso sobre } \\
\text { certas situações por } \\
\text { muito tempo, e elas } \\
\text { ainda me deixam } \\
\text { com raiva. }\end{array}$ & $\begin{array}{l}\text { Eu penso sobre } \\
\text { certos eventos } \\
\text { passados por muito } \\
\text { tempo e eles ainda } \\
\text { me chateiam. }\end{array}$ & $\begin{array}{l}\text { Eu penso sobre } \\
\text { situações que } \\
\text { aconteceram há } \\
\text { muito tempo e ainda } \\
\text { sinto raiva. }\end{array}$ & $\begin{array}{l}\text { I think about } \\
\text { situations that have } \\
\text { happened a long } \\
\text { time ago and I still } \\
\text { feel angry. }\end{array}$ & $\begin{array}{l}\text { I think about } \\
\text { situations that } \\
\text { happened a long } \\
\text { time ago and they } \\
\text { still make me angry. }\end{array}$ & $\begin{array}{l}\text { I think about } \\
\text { situations that } \\
\text { happened a long } \\
\text { time ago and they } \\
\text { still make me angry. }\end{array}$ \\
\hline $\begin{array}{l}\text { 6. I have difficulty } \\
\text { for giving } \\
\text { people who } \\
\text { have hurt me. }\end{array}$ & $\begin{array}{l}\text { Eu tenho } \\
\text { dificuldades para } \\
\text { perdoar pessoas } \\
\text { que me fizeram mal } \\
\text { (machucaram). }\end{array}$ & $\begin{array}{l}\text { Eu tenho } \\
\text { dificuldades em } \\
\text { perdoar pessoas que } \\
\text { me machucaram. }\end{array}$ & $\begin{array}{l}\text { Eu tenho dificuldade } \\
\text { para perdoar } \\
\text { pessoas que me } \\
\text { magoaram. }\end{array}$ & $\begin{array}{l}\text { I have a hard time } \\
\text { forgiving people that } \\
\text { have hurt me. }\end{array}$ & $\begin{array}{l}\text { I have a hard time } \\
\text { forgiving people that } \\
\text { hurt me. }\end{array}$ & $\begin{array}{l}\text { I have a hard time } \\
\text { forgiving people that } \\
\text { have hurt me. }\end{array}$ \\
\hline $\begin{array}{l}\text { 7. After an } \\
\text { argument is } \\
\text { over, I keep } \\
\text { fighting with } \\
\text { this person in } \\
\text { my imagination. }\end{array}$ & $\begin{array}{l}\text { Depois que uma } \\
\text { discussão foi } \\
\text { encerrada, eu } \\
\text { continuo discutindo } \\
\text { com a pessoa na } \\
\text { minha imaginação. }\end{array}$ & $\begin{array}{l}\text { Depois que uma } \\
\text { discussão acaba, eu } \\
\text { continuo brigando } \\
\text { com essa pessoa na } \\
\text { minha imaginação. }\end{array}$ & $\begin{array}{l}\text { Depois que uma } \\
\text { discussão acaba, eu } \\
\text { continuo brigando } \\
\text { com essa pessoa no } \\
\text { meu pensamento. }\end{array}$ & $\begin{array}{l}\text { After a discussion } \\
\text { ends, I keep fighting } \\
\text { with that person in } \\
\text { my mind. }\end{array}$ & $\begin{array}{l}\text { After an argument is } \\
\text { over, I keep having } \\
\text { the discussion in my } \\
\text { mind. }\end{array}$ & $\begin{array}{l}\text { After an argument is } \\
\text { over, I keep fighting } \\
\text { with that person in } \\
\text { my mind. }\end{array}$ \\
\hline $\begin{array}{l}\text { 8. Memories } \\
\text { of being } \\
\text { aggravated } \\
\text { pop up into my } \\
\text { mind before If } \\
\text { all a sleep. }\end{array}$ & $\begin{array}{l}\text { Memórias sobre } \\
\text { situações em que } \\
\text { estava irritado } \\
\text { aparecem na minha } \\
\text { mente antes de } \\
\text { dormir. }\end{array}$ & $\begin{array}{l}\text { Antes de eu } \\
\text { adormecer, tenho } \\
\text { lembranças de ser } \\
\text { maltratada. }\end{array}$ & $\begin{array}{l}\text { Antes de dormir, } \\
\text { lembro de situações } \\
\text { em que estive } \\
\text { irritado. }\end{array}$ & $\begin{array}{l}\text { Before I fall asleep, } \\
\text { I remember the } \\
\text { situations that made } \\
\text { me feel frustrated/ } \\
\text { pissed off/angry. }\end{array}$ & $\begin{array}{l}\text { Before falling } \\
\text { asleep, I remember } \\
\text { situations when I } \\
\text { was angry. }\end{array}$ & $\begin{array}{l}\text { Before I fall asleep, I } \\
\text { remember situations } \\
\text { that made me feel } \\
\text { angry. }\end{array}$ \\
\hline
\end{tabular}




\begin{tabular}{|c|c|c|c|c|c|c|c|}
\hline $\begin{array}{l}\text { Orig } \\
\text { ARS }\end{array}$ & ginal & $\begin{array}{l}\text { Translation } 1 \\
\text { (BP) }\end{array}$ & $\begin{array}{l}\text { Translation } 2 \\
\text { (BP) }\end{array}$ & $\begin{array}{l}\text { Final } \\
\text { translation (BP) }\end{array}$ & $\begin{array}{l}\text { Back- } \\
\text { translation } 1 \text { (E) }\end{array}$ & $\begin{array}{l}\text { Back- } \\
\text { translation } 2 \text { (E) }\end{array}$ & $\begin{array}{l}\text { Final Back- } \\
\text { translation (E) }\end{array}$ \\
\hline 9. & $\begin{array}{l}\text { Whenever I } \\
\text { experience } \\
\text { anger, I keep } \\
\text { thinking about } \\
\text { it for a while. }\end{array}$ & $\begin{array}{l}\text { Sempre que eu fico } \\
\text { com raiva, eu fico } \\
\text { pensando sobre isso } \\
\text { durante um certo } \\
\text { tempo. }\end{array}$ & $\begin{array}{l}\text { Sempre que sinto } \\
\text { raiva, eu fico } \\
\text { pensando sobre isso } \\
\text { por um tempo. }\end{array}$ & $\begin{array}{l}\text { Sempre que alguma } \\
\text { situação me deixa } \\
\text { com raiva, eu fico } \\
\text { pensando sobre isso } \\
\text { por um bom tempo. }\end{array}$ & $\begin{array}{l}\text { Always, when a } \\
\text { situation makes } \\
\text { me angry, I keep } \\
\text { thinking about that } \\
\text { for a long time. }\end{array}$ & $\begin{array}{l}\text { Whenever a } \\
\text { situation makes me } \\
\text { angry, I think about } \\
\text { it for a long time. }\end{array}$ & $\begin{array}{l}\text { Whenever a } \\
\text { situation makes } \\
\text { me angry, I keep } \\
\text { thinking about that } \\
\text { for a long time. }\end{array}$ \\
\hline 10. & $\begin{array}{l}\text { I have times } \\
\text { when I cannot } \\
\text { stop being } \\
\text { preoccupied } \\
\text { with a particular } \\
\text { conflict. }\end{array}$ & $\begin{array}{l}\text { Existem ocasiões } \\
\text { em que eu não } \\
\text { consigo parar de } \\
\text { me preocupar com } \\
\text { um conflito em } \\
\text { específico. }\end{array}$ & $\begin{array}{l}\text { Algumas vezes eu } \\
\text { não consigo parar } \\
\text { de me preocupar } \\
\text { sobre determinado } \\
\text { conflito. }\end{array}$ & $\begin{array}{l}\text { Eu não consigo } \\
\text { parar de me } \\
\text { preocupar com } \\
\text { uma determinada } \\
\text { situação que me } \\
\text { deixou com raiva. }\end{array}$ & $\begin{array}{l}\text { I cannot stop } \\
\text { worrying about a } \\
\text { particular situation } \\
\text { that made me } \\
\text { angry. }\end{array}$ & $\begin{array}{l}\text { I cannot stop } \\
\text { worrying over a } \\
\text { certain situation that } \\
\text { made me angry. }\end{array}$ & $\begin{array}{l}\text { I cannot stop } \\
\text { worrying about a } \\
\text { particular situation } \\
\text { that made me } \\
\text { angry. }\end{array}$ \\
\hline 11. & $\begin{array}{l}\text { I analyze } \\
\text { events that } \\
\text { make me angry. }\end{array}$ & $\begin{array}{l}\text { Eu analiso situações } \\
\text { que me deixam com } \\
\text { raiva. }\end{array}$ & $\begin{array}{l}\text { Eu avalio } \\
\text { acontecimentos que } \\
\text { me deixaram com } \\
\text { raiva. }\end{array}$ & $\begin{array}{l}\text { Eu analiso as } \\
\text { situações que me } \\
\text { deixam com raiva. }\end{array}$ & $\begin{array}{l}\text { I analyze the } \\
\text { situations that have } \\
\text { made me angry. }\end{array}$ & $\begin{array}{l}\text { I analyze situations } \\
\text { that make me angry. }\end{array}$ & $\begin{array}{l}\text { I analyze the } \\
\text { situations that make } \\
\text { me angry. }\end{array}$ \\
\hline 12. & $\begin{array}{l}\text { I think about } \\
\text { the reasons } \\
\text { people treat me } \\
\text { badly. }\end{array}$ & $\begin{array}{l}\text { Eu penso sobre os } \\
\text { motivos pelos quais } \\
\text { as pessoas me } \\
\text { tratam mal. }\end{array}$ & $\begin{array}{l}\text { Eu penso sobre as } \\
\text { razões pelas quais } \\
\text { as pessoas me } \\
\text { tratam mal. }\end{array}$ & $\begin{array}{l}\text { Quando sou } \\
\text { maltratado por } \\
\text { alguém, eu penso } \\
\text { sobre os motivos } \\
\text { pelos quais as } \\
\text { pessoas me tratam } \\
\text { mal. }\end{array}$ & $\begin{array}{l}\text { When I am } \\
\text { mistreated by } \\
\text { someone, I think } \\
\text { about the reasons } \\
\text { why people treated } \\
\text { me badly. }\end{array}$ & $\begin{array}{l}\text { Whenever I am } \\
\text { mistreated, I think } \\
\text { about the reasons } \\
\text { why people treat me } \\
\text { badly. }\end{array}$ & $\begin{array}{l}\text { When I am } \\
\text { mistreated by } \\
\text { someone, I think } \\
\text { about the reasons } \\
\text { why people treat me } \\
\text { badly. }\end{array}$ \\
\hline 13. & $\begin{array}{l}\text { I have day } \\
\text { dreams and } \\
\text { fantasies of } \\
\text { violent nature. }\end{array}$ & $\begin{array}{l}\text { Eu tenho } \\
\text { imaginações ou } \\
\text { fantasias violentas. }\end{array}$ & $\begin{array}{l}\text { Eu tenho } \\
\text { pensamentos } \\
\text { e fantasias de } \\
\text { natureza violenta. }\end{array}$ & $\begin{array}{l}\text { Eu imagino coisas e } \\
\text { tenho pensamentos } \\
\text { de conteúdo } \\
\text { violento. }\end{array}$ & $\begin{array}{l}\text { I imagine things } \\
\text { and have thoughts } \\
\text { of violence (violent } \\
\text { content). }\end{array}$ & $\begin{array}{l}\text { I imagine and have } \\
\text { thoughts of violent } \\
\text { content. }\end{array}$ & $\begin{array}{l}\text { I imagine things and } \\
\text { have thoughts of } \\
\text { violent content. }\end{array}$ \\
\hline 14. & $\begin{array}{l}\text { I feel angry } \\
\text { about certain } \\
\text { things in my } \\
\text { life. }\end{array}$ & $\begin{array}{l}\text { Eu me sinto com } \\
\text { raiva sobre algumas } \\
\text { coisas de minha } \\
\text { vida. }\end{array}$ & $\begin{array}{l}\text { Eu sinto raiva sobre } \\
\text { certas coisas em } \\
\text { minha vida. }\end{array}$ & $\begin{array}{l}\text { Eu sinto raiva de } \\
\text { certas coisas que } \\
\text { acontecem em } \\
\text { minha vida. }\end{array}$ & $\begin{array}{l}\text { I feel angry about } \\
\text { certain things that } \\
\text { have happened in } \\
\text { my life. }\end{array}$ & $\begin{array}{l}\text { I feel angry about } \\
\text { certain events that } \\
\text { happen in my life. }\end{array}$ & $\begin{array}{l}\text { I feel angry about } \\
\text { certain things that } \\
\text { happen in my life. }\end{array}$ \\
\hline 15. & $\begin{array}{l}\text { When someone } \\
\text { makes me } \\
\text { angry, I can't } \\
\text { stop think in } \\
\text { gab out how to } \\
\text { get back at this } \\
\text { person. }\end{array}$ & $\begin{array}{l}\text { Quando alguém me } \\
\text { deixa com raiva, eu } \\
\text { não consigo parar de } \\
\text { pensar sobre como } \\
\text { posso me vingar } \\
\text { dessa pessoa. }\end{array}$ & $\begin{array}{l}\text { Quando alguém me } \\
\text { faz raiva, eu não } \\
\text { consigo parar de } \\
\text { pensar sobre como } \\
\text { eu poderia dar uma } \\
\text { devolutiva à essa } \\
\text { pessoa. }\end{array}$ & $\begin{array}{l}\text { Quando alguém me } \\
\text { deixa com raiva, eu } \\
\text { não consigo parar } \\
\text { de pensar sobre } \\
\text { como eu poderia me } \\
\text { vingar dessa pessoa. }\end{array}$ & $\begin{array}{l}\text { When someone } \\
\text { makes me angry, I } \\
\text { cannot stop thinking } \\
\text { about how I could } \\
\text { get back on that } \\
\text { person. }\end{array}$ & $\begin{array}{l}\text { When someone } \\
\text { makes me mad, I } \\
\text { cannot stop thinking } \\
\text { about how I could } \\
\text { get back on them. }\end{array}$ & $\begin{array}{l}\text { When someone } \\
\text { makes me angry, I } \\
\text { cannot stop thinking } \\
\text { about how I could } \\
\text { take get back on } \\
\text { that person. }\end{array}$ \\
\hline 16. & $\begin{array}{l}\text { When someone } \\
\text { provokes me, I } \\
\text { keep wondering } \\
\text { why this should } \\
\text { have happened } \\
\text { to me. }\end{array}$ & $\begin{array}{l}\text { Quando alguém } \\
\text { me provoca, fico } \\
\text { pensando porque } \\
\text { essas coisas } \\
\text { acontecem comigo. }\end{array}$ & $\begin{array}{l}\text { Quando alguém me } \\
\text { provoca, eu fico } \\
\text { pensando porque } \\
\text { isto deveria ter } \\
\text { acontecido comigo. }\end{array}$ & $\begin{array}{l}\text { Quando alguém } \\
\text { me provoca, fico } \\
\text { pensando em } \\
\text { porque essas } \\
\text { coisas tinham que } \\
\text { acontecer comigo. }\end{array}$ & $\begin{array}{l}\text { When someone } \\
\text { provokes me, I keep } \\
\text { thinking in why } \\
\text { those things have to } \\
\text { happen with me. }\end{array}$ & $\begin{array}{l}\text { When someone } \\
\text { teases me, I think } \\
\text { about why these } \\
\text { things had to } \\
\text { happen to me. }\end{array}$ & $\begin{array}{l}\text { When someone } \\
\text { provokes me, I keep } \\
\text { thinking in why } \\
\text { those things had to } \\
\text { happen to me. }\end{array}$ \\
\hline 17. & $\begin{array}{l}\text { Memories of } \\
\text { even minor } \\
\text { annoyances } \\
\text { bother me for a } \\
\text { while. }\end{array}$ & $\begin{array}{l}\text { Memórias } \\
\text { de pequenas } \\
\text { chateações me } \\
\text { incomodam por um } \\
\text { certo tempo. }\end{array}$ & $\begin{array}{l}\text { Mesmo } \\
\text { acontecimentos } \\
\text { pequenos ficam na } \\
\text { minha memória me } \\
\text { chateando por um } \\
\text { tempo. }\end{array}$ & $\begin{array}{l}\text { Lembranças } \\
\text { de pequenas } \\
\text { chateações me } \\
\text { incomodam por um } \\
\text { bom tempo. }\end{array}$ & $\begin{array}{l}\text { Memories of } \\
\text { small annoyances } \\
\text { (problems) bother } \\
\text { me for quite a while. }\end{array}$ & $\begin{array}{l}\text { Memories of small } \\
\text { annoyances bother } \\
\text { me for a long time. }\end{array}$ & $\begin{array}{l}\text { Memories of small } \\
\text { annoyances bother } \\
\text { me for quite a while. }\end{array}$ \\
\hline 18. & $\begin{array}{l}\text { When } \\
\text { something } \\
\text { makes me } \\
\text { angry, I turn } \\
\text { this matter over } \\
\text { and over again } \\
\text { in my mind. }\end{array}$ & $\begin{array}{l}\text { Quando alguma } \\
\text { coisa me deixa com } \\
\text { raiva, eu penso } \\
\text { sobre isso várias } \\
\text { vezes. }\end{array}$ & $\begin{array}{l}\text { Quando algo me } \\
\text { chateia, eu trago } \\
\text { esse assunto na } \\
\text { minha cabeça } \\
\text { repetidas vezes. }\end{array}$ & $\begin{array}{l}\text { Quando alguma } \\
\text { coisa me deixa } \\
\text { com raiva, eu fico } \\
\text { pensando sobre } \\
\text { esse assunto várias } \\
\text { vezes. }\end{array}$ & $\begin{array}{l}\text { When something } \\
\text { makes me feel } \\
\text { angry, I keep } \\
\text { thinking about it } \\
\text { several times. }\end{array}$ & $\begin{array}{l}\text { When something } \\
\text { makes me angry, } \\
\text { I think about that } \\
\text { many times. }\end{array}$ & $\begin{array}{l}\text { When something } \\
\text { makes me angry, I } \\
\text { keep thinking about } \\
\text { it many times. }\end{array}$ \\
\hline 19. & $\begin{array}{l}\text { I re-enact the } \\
\text { anger episode } \\
\text { in my mind } \\
\text { after it has } \\
\text { happened. }\end{array}$ & $\begin{array}{l}\text { Eu enceno } \\
\text { novamente o } \\
\text { episódio que me } \\
\text { deixou com raiva } \\
\text { na minha cabeça } \\
\text { quando ele já } \\
\text { acabou. }\end{array}$ & $\begin{array}{l}\text { Eu revivo episódios } \\
\text { de raiva na minha } \\
\text { memória mesmo } \\
\text { depois que o evento } \\
\text { aconteceu. }\end{array}$ & $\begin{array}{l}\text { Eu relembro um } \\
\text { momento de raiva } \\
\text { na minha cabeça } \\
\text { depois que ele } \\
\text { aconteceu. }\end{array}$ & $\begin{array}{l}\text { I keep remembering } \\
\text { a moment of anger } \\
\text { in my head after it } \\
\text { happened. }\end{array}$ & $\begin{array}{l}\text { I recall a moment } \\
\text { of anger after it } \\
\text { happened. }\end{array}$ & $\begin{array}{l}\text { I recall a moment } \\
\text { of anger in my mind } \\
\text { after it happened. }\end{array}$ \\
\hline $1-$ & Almost never & Quase nunca & Quase nunca & Quase nunca & Almost never (never) & Hardly ever & Almost never \\
\hline $2-$ & Sometimes & Às vezes & Às vezes & Às vezes & Sometimes & Sometimes & Sometimes \\
\hline $3-$ & Often & Com frequência & Frequentemente & Muitas vezes & Very often & Frequently & Frequently \\
\hline $4-$ & Almost always & Quase sempre & Quase sempre & Quase sempre & $\begin{array}{l}\text { Almost always } \\
\text { (always) }\end{array}$ & Almost always & Almost always \\
\hline
\end{tabular}

ARS = Anger Rumination Scale $; \mathrm{BP}=$ Brazilian Portuguese $; \mathrm{E}=$ English . 
This consideration was taken into account and this item was edited as follows: "Eu penso muito sobre momentos passados em que tive raiva," replacing the term "ruminar" (to ruminate) with "penso muito" (over think, think too much). Another term was also identified by the experts as misleading in the Brazilian context: "I have daydreams and fantasies of violent nature." There is no perfect translation to the term "daydreams" in Portuguese, therefore "I have daydreams" was translated as "Eu imagino" (I imagine). Also, the term "fantasies" can be interpreted in Brazilian Portuguese in different ways, and thus it was replaced with "pensamentos," as follows: "Eu imagino coisas e tenho pensamentos de conteúdo violento." Finally, the experts suggested the inclusion of a limiter of time "in the last 6 months" in the questionnaire's instructions. After discussion, they judged that the definition of "in the last six months" would not change the original construct and would facilitate understanding in the Brazilian context.

\section{Translation and back-translation}

The forward-and back-translations followed the steps described above, involving six translators throughout the process. Few items showed discrepancies between the versions of the two independent translators and the two back-translators, which facilitated the task of the translators responsible for synthesizing the versions. The expert committee checked the synthesized forward- and back-translations, comparing them to the original ARS. The committee observed that the words "anger" and "angry," used in many items of the original instrument, were randomly translated into Portuguese as "raiva" and "chatear," not following a pattern. The same happened with the word "rumination," which was translated as "penso muito" and "rumino." After discussing this issue, adjustments were performed and a decision was reached about the final wording of items in the ARS-Brazil. Table 1 shows the original items of the ARS, results of the translation and back-translation stages, and the final version of the Brazilian Portuguese correlate items after the pilot study and review by the expert committee.

\section{Pretest}

The mean age of participants was 35.3 years (standard deviation $=15.96), 64.7 \%$ were women. Level of education was high school or lower in $36 \%$ and college or higher in $64 \%$. The mean level of clarity of the questions was very high, ranging from 84.3 to $100 \%$ of understanding. Of all 49 participants, only two had difficulties understanding two distinct items (each of these two participants did not understand one specific item): item 7 - "Depois que uma discussão acaba, eu continuo brigando com essa pessoa no meu pensamento"; and item 11 - "Eu analiso as situações que me deixam com raiva." None of these participants made suggestions to change the items, and none of the other 47 participants suggested changes to such items. After evaluation of the experts, it was decided to respectively modify them to "Depois que uma discussão acaba, eu continuo imaginando brigas com essa pessoa no meu pensamento"; and "Eu tento entender as situações que me deixam com raiva."

The items with the highest number of suggestions for clarity were items 1 and 10, respectively, "Eu penso muito sobre minhas experiências passadas de raiva" and "Algumas vezes eu não consigo parar de me preocupar com um determinado conflito." Nine participants suggested changes in item 1 , and eight in item 10 . The number of participants who answered "fully understood" was $43(87.75 \%)$ for item 1 and $41(83.67 \%)$ for item 10 (no one answered "I did not understand it" to such items).

For item 1, some of the suggestions were that the expression "experiências passadas de raiva" would be too formal, and the use of "situações/experiências no passado em que senti raiva" was suggested. Another participant suggested "Eu penso muito nos momentos que fiquei com muita raiva." This item was then modified by the expert committee to "Eu penso muito sobre momentos passados em que tive raiva." As for item 10, some participants suggested removing the word "algumas vezes" from the question, and others suggested replacing the word "conflito" because they judged this was not a clear question. This item was changed by the experts to "Eu não consigo parar de me preocupar com uma determinada situação que me deixou com raiva."

\section{Investigation of operational equivalence}

There were no sources of difficulty regarding the format, instructions, method of assessment, or measurement methods of the ARS in the Brazilian context. The review of the literature also demonstrated that many of the instruments used for assessing anger symptoms in Brazilian adults follow operational procedures similar to those of the ARS, e.g., the Brazilian version of the State-Trait Anger Expression Inventory (STAXI). ${ }^{16}$

\section{Discussion}

Here we presented the Brazilian version of the ARS. The scale showed proper conceptual and item equivalence, translation and back-translation 
procedures were performed adequately, and the scale demonstrated good levels of clarity among participants, as well as operational equivalence.

The ARS-Brazil is presented as a new instrument now available for the assessment of anger rumination symptoms. The scale can be used in community settings, serving as a screening tool to identify people at risk for developing maladjustment behaviors because of anger symptoms, assisting in preventive interventions. It can also be used in academic settings in studies designed to assess anger rumination indicators or symptoms in terms of their frequency, severity, or structure. Finally, another possible application of the ARS-Brazil is its use in clinical settings, as an auxiliary tool for diagnostic and therapeutic evaluations regarding the structure and severity of diseases linked to anger symptoms.

The ARS-Brazil is now among the few scales, such as the STAXI, which is available for assessing anger in Brazilian individuals. ${ }^{17}$ Nonetheless, the ARS focuses specifically on the cognitive processes of rumination, while the STAXI assesses broad aspects of anger as a state and personality trait of individuals. ${ }^{16}$ As described in previous studies, anger and anger rumination are different cognitive processes, and the Brazilian literature, so far, lacked an instrument designed to address anger rumination thoroughly. Anger rumination covers not only the aspects of irritability and anger per se (as measured by the STAXI), but also the detailed and fine-grained cognitive processes of angry afterthoughts, angry memories, thoughts of revenge and understanding of causes - the very foundations of anger rumination. ${ }^{2}$

Some limitations to this work should be noted. First, even with the attempt by the authors to prioritize participants with lower socioeconomic level and less educated, clarity levels were mainly investigated with a very selected sample, consisting of a majority of participants with higher education, which do not represent the Brazilian population. Second, and also very important, the pretest was conducted in just one site in Brazil, making national validation assurance difficult given the cultural and linguistic variances between different parts of Brazil. Nevertheless, this pretest was mainly focused on investigating suggestions for the translation process and on detecting important clarity issues. Despite that, further studies should be investigated with larger and diverse samples to assure the appropriateness of the ARS properties to the Brazilian population.

The ARS-Brazil seems to be very similar to the original ARS, suggesting that future cross-cultural studies may benefit from this early version. However, other studies are needed in order to take on further steps in the cross-cultural adaptation process of the ARS-Brazil. For example, next steps could include administering the scale to Brazilian samples, so as to collect evidence of instrument validity based on psychometric properties of the ARS-Brazil through recognized statistical methods. ${ }^{11,13,18}$

\section{Acknowledgements}

This project was funded by Conselho Nacional de Desenvolvimento Científico e Tecnológico (CNPq) and Fundo de Incentivo à Pesquisa e Eventos - Hospital de Clínicas de Porto Alegre (FIPE-HCPA).

We would like to thank Dr. Denis G. Sukhodolsky for generously approving the scale translation developed by him (ARS) for the Brazilian Portuguese version. We are also grateful to Drs. André Luiz Moreno, Bruno Figueiredo Damásio and Susana Núñez Rodriguez for their important contribution to ARS-Brazil's translation and back-translation process.

\section{Disclosure}

No conflicts of interest declared concerning the publication of this article.

\section{References}

1. Lerner JS, Li Y, Valdesolo P, Kassam KS. Emotion and decision making. Psychology. 2015;66:799-823.

2. Sukhodolsky DG, Golub A, Cromwell EN. Development and validation of the Anger Rumination Scale. Personal Individ Differ. 2001;31:689-700.

3. Andrade NO, Alcázar-Olán R, Matías OM, Guerrero AR, Espinosa AD. Anger Rumination Scale: validation in Mexico. Span J Psychol. 2017;20:1-9.

4. Martin R, Watson D, Wan CK. A three-factor model of trait anger: dimensions of affect, behavior, and cognition. J Pers. 2000;68:869-97.

5. Wilkowski BM, Robinson MD. The cognitive basis of trait anger and reactive aggression: an integrative analysis. Personal Soc Psychol Rev. 2008;12:3-21.

6. Ramos-Cejudo J, Salguero JM, Kannis-Dymand L, García-Sancho E, Love S. Anger rumination in Australia and Spain: validation of the Anger Rumination Scale. Aust J Psychol. 2017;69:293-302.

7. Uceda IM, Bleda JHL, Nieto MÁP, Sukhodolsky DG, Martínez AE. Psychometric properties of the Spanish adaptation of the Anger Rumination Scale: evidence of reliability and validity in the general population. Span J Psychol. 2016;19:E17.

8. Besharat MA. Factorial and cross-cultural validity of a Farsi version of the Anger Rumination Scale. Psychol Rep. 2011;108:317-28.

9. Maxwell JP, Moores E, Chow CCF. Anger rumination and selfreported aggression amongst British and Hong Kong Chinese athletes: a cross cultural comparison. Int J Sport Exerc Psychol. 2007; 5:9-27.

10. Reynes E, Berthouze-Aranda SE, Guillet-Descas E, Chabaud P, Deflandre A. Validation française de l'Échelle de Rumination de Colère (ARS). L'Encéphale. 2013;39:339-46.

11. Gjersing L, Caplehorn JR, Clausen T. Cross-cultural adaptation of research instruments: language, setting, time and statistical considerations. BMC Med Res Methodol. 2010;10:1-10. 
12. Reichenheim ME, Moraes CL. Operationalizing the cross-cultural adaptation of epidemological measurement instruments. Rev Saude Publica. 2007;41:665-73.

13. International Test Commission. International Test Commission guidelines for translating and adapting tests. 2nd ed. Version 2.3. 2016. [cited 01 Mar 2017]. http://www.intestcom.org/files/ guideline_test_adaptation_2ed.pdf.

14. Beaton DE, Bombardier C, Guillemin F, Ferraz MB. Guidelines for the process of cross-cultural adaptation of self-report measures. Spine. 2000;25:3186-91.

15. Wang $W-L$, Lee $H-L$, Fetzer $S J$. Challenges and strategies of instrument translation. West J Nurs Res. 2006;28:310-21.

16. Spielberger $C D$. Inventário de Expressão de Raiva como Estado e Traço (STAXI): Manual Técnico. Porto Alegre: Vetor; 1992.
17. Azevedo FB de, Wang Y-P, Goulart AC, Lotufo PA, Benseñor IM. Application of the Spielberger's State-Trait Anger Expression Inventory in clinical patients. Arq Neuropsiquiatr. 2010;68:231-4. 18. Guillemin F, Bombardier C, Beaton D. Cross-cultural adaptation of health-related quality of life measures: literature review and proposed guidelines. J Clin Epidemiol. 1993;46:1417-32.

\section{Correspondence:}

Daniela Sperotto

Centro de Pesquisa Clínica (CPC), $6^{\circ}$ andar

Rua Ramiro Barcelos, 2350, Santa Cecília

90035-007 - Porto Alegre, RS - Brazil

Tel./Fax: +55 (51) 33598943

E-mail: daniela_sperotto@yahoo.com.br 